\title{
PISO BLANDO, UNA FALLA COMÚN EN EL TERREMOTO DE PEDERNALES
}

\section{SOFT.STORY, A COMMON FLAW IN THE EARTHQUAKE IN PEDERNALESS}

\author{
Dario Páez $^{(1)}$, José Zabala(2), Yuri Rodríguez ${ }^{(1)}$ \\ ${ }^{(1)}$ Facultad de Ingeniería \\ Universidad Laica Eloy Alfaro de Manabí \\ Ciudadela Universitaria, Vía San Mateo, Manta, Ecuador \\ julio.paez@uleam.edu.ec \\ yuri.rodriguez@uleam.edu.ec \\ ${ }^{(2)}$ Facultad de Ingeniería \\ Universidad del Zulia \\ Avenida 16 y calle $67 \mathrm{~B}$, Maracaibo, Venezuela \\ izabala@fing.luz.edu.ve

\section{RESUMEN}

Este artículo estudia los daños que las edificaciones han sufrido ante diferentes sismos importantes de América en las últimas décadas, caracterizando los eventos de manera local y las repercusiones que ha tenido en relación a su impacto. Se focaliza el estudio de daño en estructuras con tipo piso débil o blando, las mismas que se caracterizan por la diferencia de resistencia, rigidez y ductilidad entre los niveles de una edificación.

Esta realidad en el desempeño de las edificaciones durante el tiempo, se la compara con el alto porcentaje de colapso por piso blando de edificaciones por el terremoto de Muisne del 16 de abril del 2016, categorizada como una falla común y causa del colapso de muchas edificaciones. Por último se plantea diversas soluciones para evitar este tipo de falla.

Palabras clave: Piso blando - diseño sismo resistente - rigidez - colapso de edificio

\section{ABSTRACT}

This article evidences the damage that the buildings have suffered in different important earthquakes in America in the last decades, characterizing the events locally in relation to its impact. The study focuses on damage structures with weak or flexible type flats; they are characterized by the difference of strength, stiffness and ductility among the levels of a building.

This fact in the performance of the buildings during the period is compared with the high percentage of collapsed buildings in the earthquake of Muisne in April $16^{\text {th }}, 2016$, categorized as a common failure and cause of the collapse of many buildings. Finally, various solutions are suggested to avoid this type of failure. 
Keywords: Soft-story - seismic-resistant - stiffness - building collapse

\section{INTRODUCCIÓN}

La caracterización de las estructuras en el Ecuador, en función del peligro sísmico al que se encuentra expuesto el país y la región por su ubicación, resulta de suma importancia para poder identificar los posibles daños que se esperan que se presenten en las edificaciones, en el supuesto caso de que se encuentren expuestas ante la arremetida de un sismo de características superiores al considerado al momento del diseño. Razón por la cual, la Norma Ecuatoriana de Construcción establece diferentes niveles de seguridad, donde se indica cómo sismo de diseño el nivel de seguridad de vida, entendiéndose que se debe proteger la vida de quienes se encuentren en las edificaciones ante un terremoto de 475 años de período de retorno y de igual forma, impedir el colapso total ante un sismo de gran magnitud de 2500 años de periodo de retorno, este último para estructuras de ocupación esencial y estructuras especiales. Alcanzándose esto, cuando se diseña para las fuerzas máximas esperadas, controlándose los desplazamientos relativos generales y utilizándose criterios de disipación de energía inelástica de acuerdo a principios básicos, que hoy en día incorpora cualquier filosofía de diseño sismo resistente para las estructuras de las edificaciones en Ecuador.

A pesar de la frecuencia de terremotos acontecidos a nivel mundial, resulta difícil afirmar, que después de la ocurrencia del mismo, las personas no "aprendan la lección", a pesar de las grandes pérdidas de vida y materiales que este tipo de evento deja en una comunidad, ni aun así, por el grado de afectación que sufre una determinada población, la cual se circunscribe al nivel de preparación de sus habitantes dentro del ámbito económico, psicológico, social y demográfico, siendo estos proporcionales a la intensidad del evento en la extensión geográfica que ocupa la comunidad.

Las experiencias sísmicas existentes en Ecuador, como en el resto del mundo, han dejado muy claro, que la "memoria frágil" ha jugado un papel fundamental en la preparación ante este tipo de desastres naturales, siendo así, que al comparar la respuesta de las estructuras ante la protección de la población, objetivo de éste estudio, evidencian la existencia de mucha similitud con los daños colaterales que estos dejan en otros lugares de ocurrencia de terremotos.

Esta investigación se plantea analizar las fallas de las estructuras con piso blando o flexible en diferentes eventos sísmicos ocurridos a nivel de América, detallando de manera general las características de los eventos, con la finalidad de contrastar los daños que el terremoto del 16 de abril de 2016, dejó en MuisneEcuador, evidenciándose similitudes y semejanzas a nivel de falla y daños.

\section{DEFINICIÓN PISO BLANDO}

El piso blando se origina generalmente, cuando se cuenta con un entrepiso en el cual su rigidez es considerablemente menor a la de los niveles subyacentes y 
adyacentes, los modos de vibrar de la estructura, así como la respuesta general, en comparación con una estructura regular en altura, son diferentes (Fernández, 2007).

Uno de los principios básicos en el diseño de estructuras sismo resistentes es que exista una cierta configuración cuadrada, circular u ovalada en la forma de la distribución en planta y una regularidad en su elevación lo más cercano a la rectangularidad de la edificación, lo ideal es que ésta, presente una simetría tanto en forma, cómo en volumen. Siendo esto una condición, para que la estructura se comporte estructuralmente de una manera correcta, ante las fuerzas inducidas por el sismo, ya que si no se cumple con este principio, la edificación corre el riesgo de tener problemas, tales como el colapso parcial o total de los elementos estructurales, en el peor de los casos.

Una problemática frecuente que se ha podido apreciar en las últimas décadas en la construcción de las edificaciones, han sido fallas de estructuras por tener en su diseño piso blando, donde un elemento estructural cesa su desempeño y deja de funcionar en forma satisfactoria su capacidad resistente (Kaminetzky, 1991).

Se denomina piso blando en aquellos edificios, cuya planta baja está diseñada y destinada para parqueadero vehicular, restaurantes, áreas verdes, lobbies, entre otras; de tal forma, que sólo existen elementos verticales o columnas a este nivel y en los pisos superiores, se construyen cerramientos para viviendas con mampostería, armada o no, lo que hace estructuralmente es que la planta baja tenga menor rigidez que las pisos superiores, cuando lo sísmico resistente correcto, es lo contrario, mayor rigidez en las plantas inferiores.

El diseño y uso de éste piso blando, es una práctica común que realizan los arquitectos buscando soluciones de espacios, ya que resulta muy beneficioso y rentable, tener esos espacios amplios en la parte baja del edificio, con el fin de ubicar restaurantes, sala de eventos, parqueaderos, entre otros. Aunque desde el punto de vista sismo resistente, no es recomendado usarlos, y debido a esto, las normas sismo resistentes de diferentes países que están en peligro sísmico, se están adaptando a mejorar y buscar la manera de evitar estos fallos (Fig. 1) en el diseño de las edificaciones.

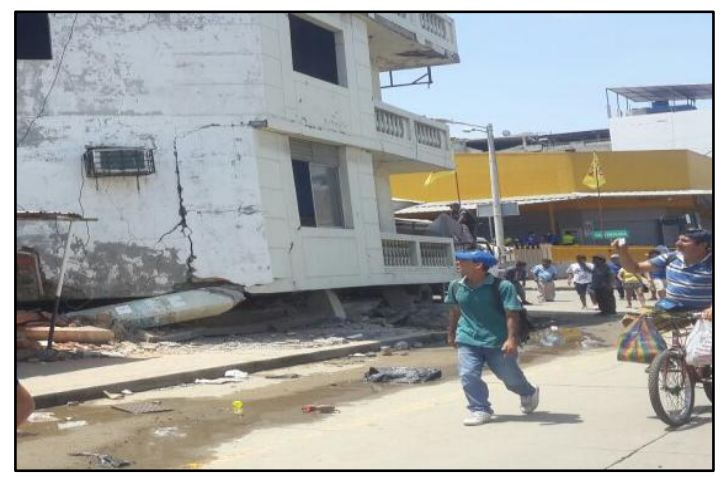

Figura 1. Estructura colapsada por falla de piso blando 
Es importante indicar, que la Norma Ecuatoriana de la Construcción (NEC, 2015), en el capítulo concerniente de Peligro Sísmico, define como Piso Blando en una edificación, cuando esta presenta una rigidez lateral en un piso inferior, menor al $70 \%$ de la rigidez lateral del piso inmediato superior. Y cómo Piso Débil, cuando su resistencia lateral del piso inferior es menor al $80 \%$ de la resistencia lateral del piso inmediato superior.

La configuración estructural en el diseño de las edificaciones es un factor muy importante ante la presencia de los embates de una acción o fuerza sísmica, ya que el desempeño resistente del edificio, tiene como una variable muy importante, su regularidad en planta y elevación. Últimamente, se ha podido evidenciar que en edificaciones irregulares expuestas a grandes fuerzas sísmicas, éstas en varias ocasiones llegan a presentar daños graves, hasta incluso, la pérdida de estabilidad estructural.

En razón de lo anterior, existen varios factores que deben considerase al momento de la estructuración, siendo entre estos: la resistencia, la rigidez y la distribución de la masa o cargas, los cuales deben tener un diseño y una disposición en lo posible correctamente equilibrada y homologada.

Se puede establecer, que las irregularidades en altura se entienden como cambios bruscos de la rigidez entre pisos continuos, lo que origina que durante la presencia del terremoto, los esfuerzos por disipar la energía sísmica del input, se vean focalizados en los elementos estructurales, principalmente en las columnas, que definen los pisos blandos. (Pujades, 2003)

Durante un terremoto, las fuerzas laterales del mismo que actúan en la estructura, no pueden ser bien distribuidas a lo largo de la altura de la edificación. Por lo que esta situación, hace que las fuerzas laterales sísmicas tiendan a concentrarse en los pisos, teniendo éstos un gran desplazamiento. Lo que forma entonces en las uniones columna/piso, un mecanismo de fallo, que puede llevar al colapso del sistema estructural, sumado al alto nivel de efecto de las cargas gravitacionales, como se muestra en la Figura 2, la cual muestra un ensayo en laboratorio de este tipo de falla estructural por presencia de piso débil.

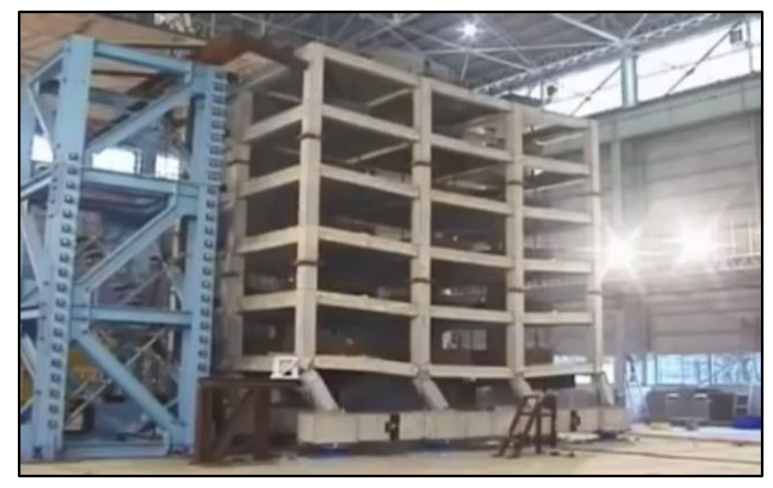

Figura 2. Ensayo de falla de piso débil en primer nivel 
Según Granizo (2013), el piso blando o débil se puede presentar cuando existe un cambio considerable de rigidez entre los pisos continuos, que se puede deber a diferencia de altura de las columnas o a la interrupción de elementos de encerramiento, como la mampostería.

La interacción sismo-estructura es un campo de estudio para conocer, cómo será el desempeño de la estructura, el cual se inicia, cuando la fuerza sísmica es trasmitida a la estructura por efecto de las ondas elásticas al chocar contra la cimentación, donde la energía que se transmite desde el subsuelo, asciende hasta la cresta del edificio, provocando el efecto de resonancia, induciendo con esto un desplazamiento, luego esta energía es disipada y el edificio vuelve a su posición original. Ahora, si la estructura cuenta con piso blando en la planta baja, y dependiendo de la magnitud del sismo y las propiedades del suelo, surgirá un exceso de esfuerzo provocando la rotura en cabeza de columna, lo que llevará a la edificación al colapso, como se muestra en la Figura 3.

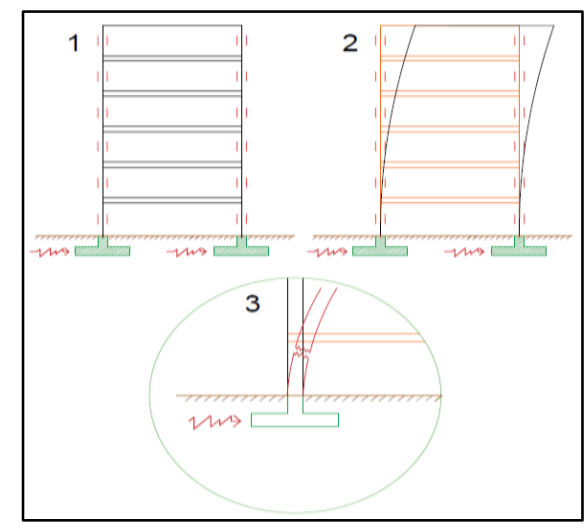

Figura 3. Comportamiento de colapso de piso débil

En cambio, si el sismo es de gran intensidad, se produce en el piso blando un fuerte movimiento lateral, como se puede apreciar en la figura 4, lo que aumenta la carga vertical $\mathrm{P}$ producida por los pisos superiores de la estructura, con lo que se ejercen presión sobre el primer piso, y a su vez, se genera un momento M que debe ser equilibrado, de lo contrario colapsará la estructura.

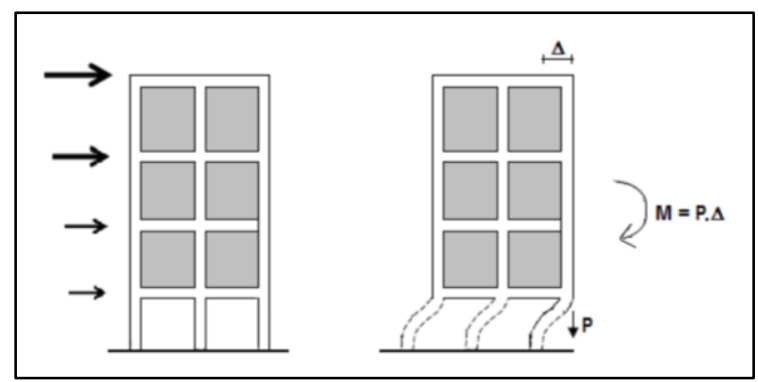

Figura 4. Efecto sobre piso blando 
Entonces, esta acción produce un volque de la estructura hacia su parte más débil, y se produce una rótula plástica entre viga-columna producidas por el momento ejercido.

Se ha observado, que ante movimientos sísmicos de intensidades considerables, aquellas edificaciones que carecen de irregularidades en su estructura, tienen mejor comportamiento que aquellas estructuras donde existen secciones irregulares, pudiéndose confirmar esta afirmación, al comparar aquellos edificios que colapsaron con los que lograron mantenerse en pie. Por lo que es evidente que las estructuras que resistieron en pie, la eventualidad sísmica del 16 de abril del 2016, contaban con una regularidad en toda su estructura.

El comportamiento de las estructuras irregulares es difícil de predecir y por tanto, los métodos de diseño resultan de reducida confiabilidad, razón por la cual, se opta por aumentar las fuerzas de diseño. Esto se logra usando un factor de reducción menor de fuerza sísmica R. (Quincot, 2010)

Muchas veces se diseñan las columnas del piso blando para soportar los momentos flectores (Mf) producidos por el movimiento telúrico. Pero se omiten los momentos producidos por el desplazamiento lateral $\mathrm{M}$, ante un sismo, el momento flector que actúa $M=M f+M$ supera la capacidad del momento flector Mf, lo que da lugar al fenómeno de rotula plástica en los extremos de las columnas del piso blando, lo que vuelve flexible a esta parte de la estructura aumentando $M, y$ permitiendo el colapso de la estructura.

\section{FALLA COMÚN DE COLAPSO EN EVENTOS SÍSMICOS MUNDIALES}

Se plantea en esta sección, la revisión de algunos eventos sismicos que han sido considerados importantes por su capacidad de destrucción, donde de acuerdo al análisis realizado por los autores, el colapso de los mismos o un porcentaje considerable de daños, es atribuible a falla por piso blando.

\subsection{Sismo de Valparaíso, Chile (1985)}

Es conocido que Chile presenta una sismicidad activa considerable y el terremoto de Valparaíso tuvo una intensidad de 7,8 en la escala de Richter, con una aproximación de 180 fallecidos, 260 heridos, 150.000 edificaciones destruidas y un millón de personas dignificadas.

Los daños que se dieron en relación a este evento, fueron considerados una lección importante para la ingeniería sismo resistente, que para el momento estaba en un desarrollo importante. Las edificaciones que presentaban irregularidad en elevación, así como en planta, sufrieron muchos daños, como es el caso del edificio que se muestra en la figura 5, ubicado en la ciudad de Valparaíso, que por su situación de esbeltez en columnas de primer piso, presentó falla por piso débil. 

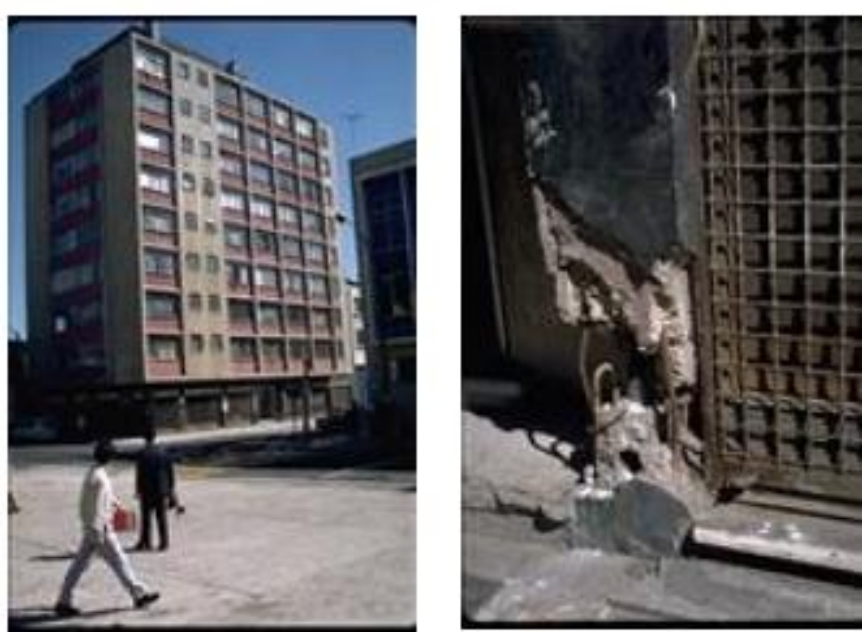

Figura 5. Efecto sobre piso blando

\subsection{Sismo del Salvador (1986)}

Este sismo de categoría superficial con una magnitud $\mathrm{Mb}=5.4$, ocurrió el 10 de octubre de 1986, en el cual fallecieron más 2.000 habitantes, diez mil heridos y unas 60.000 edificaciones destruidas (Álvarez, 1987), el cual fue categorizada como una "hecatombe" por la prensa nacional.

Nuevamente, producto de la diferencias de rigideces entre pisos, se puede apreciar en la edificación de la figura 6 , cómo se generó la falla por planta débil.

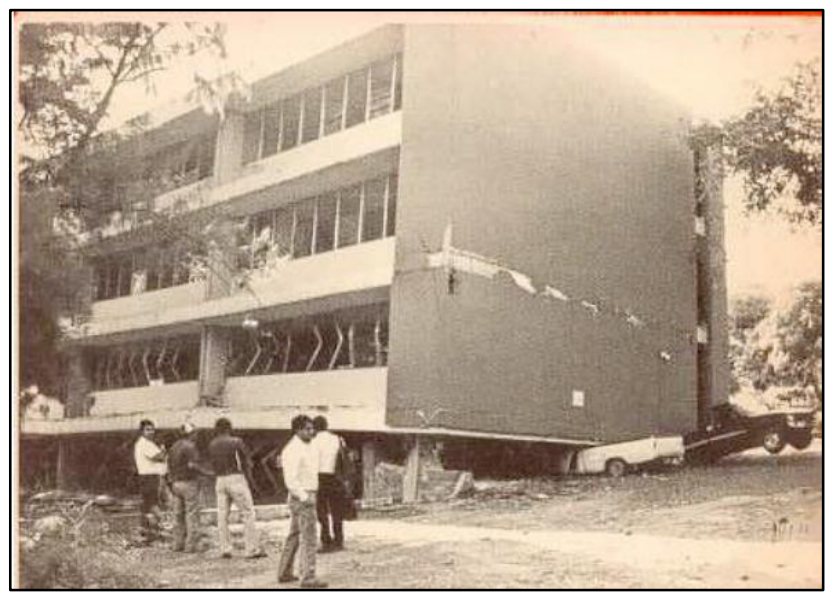

Figura 6. Daño en la estructura

Pudiéndose observar que la planta baja, aparentemente diseñado para estacionamiento vehicular, se encuentra aplastada por el resto de la edificación; a 
pesar del que el edificio estaba diseñado de manera aporticada y con muros rígidos en los pisos superiores.

\subsection{Sismo de San Fernando, Estados Unidos (1971)}

Considerado como uno de los terremotos más trágicos en los Estados Unidos de Norteamérica, ocurrido el 9 de febrero de 1971 con una magnitud de 6.6, y que debido a su fuerza de destrucción, originó daño en dos hospitales.

Una de la casa asistencial que tuvo mayor daño, fue el hospital Olive View, que constaba de 5 pisos más un sótano, estructura que presentaba grandes masas con discontinuidades considerables en su elevación y se adicionaba la irregularidad en planta; una de las secciones del edificio, planta baja, tenía piso blando, situación que ha sido considerado como uno de los casos más estudiados dentro del área de diseño de edificaciones hospitalarias. A continuación se presenta una serie de fotografías (Figura 7), donde se muestran los daños indicados.

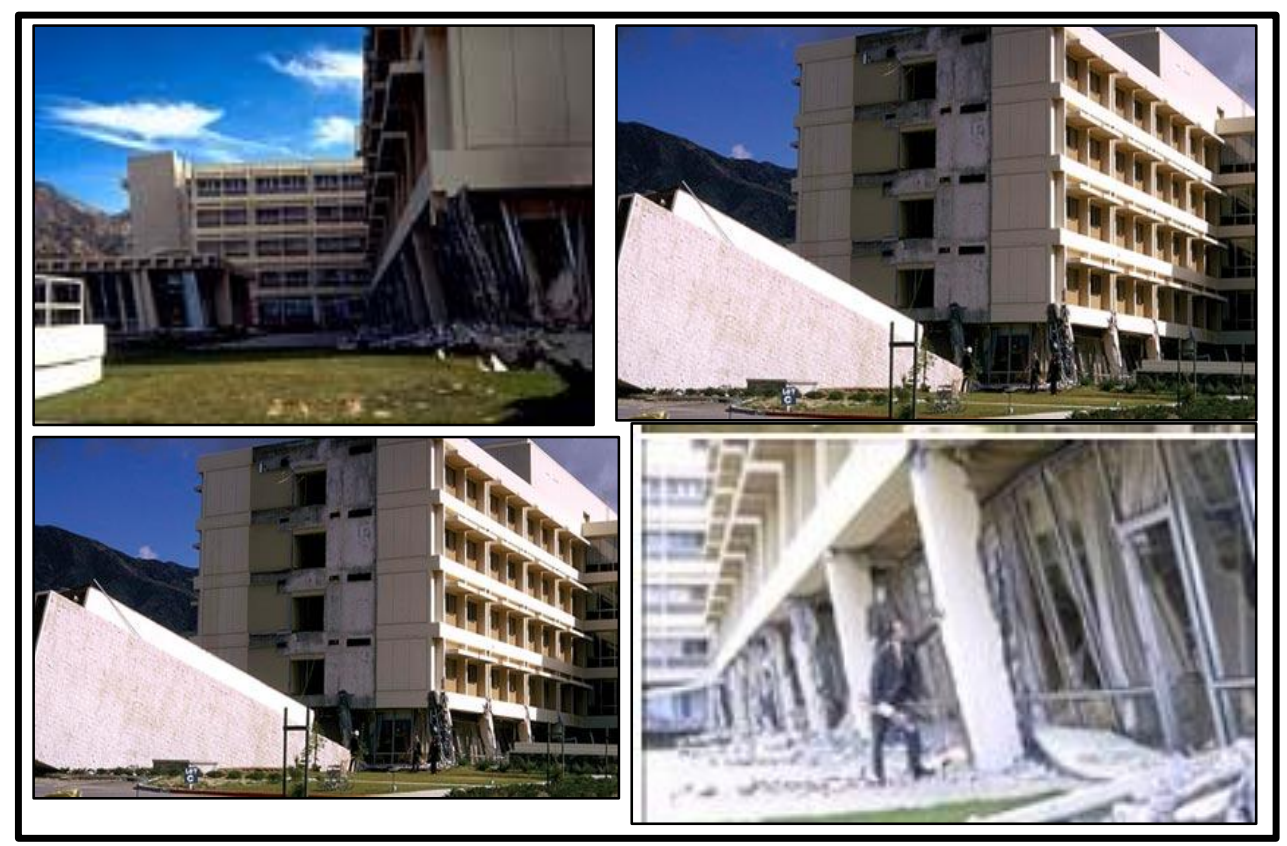

Figura 7. Hospital Olive View con falla de piso flexible

Las diferencias que se presentaron a nivel de resistencia, ductilidad y rigidez en los elementos estructurales, así como la presencia de masas que no tenían mayor importancia estructural, fue la causa por la cual el desempeño estructural haya sido poco adecuado para resistir las fuerzas sísmicas originadas por este sismo. 


\subsection{Sismo Michoacán, México (1985)}

Ocurrido el jueves 19 de septiembre de 1985, y conocido como el terremoto México del 85, con este evento se registró los mayores daños en la zona centro, sur y occidental, y fue categorizado como el más mortífero de la historia sísmica de este país. La duración y magnitud fue de 2 minutos 15 segundos y $8,1 \mathrm{Mw}$, respectivamente. El epicentro fue determinado en el estado de Michoacán, con características de trepidatorio y oscilatorio con una profundidad de $15 \mathrm{Km}$.

Las fallas en las edificaciones por irregularidad a causa de este sismo, fueron muy importantes, aun cuando estas edificaciones se encontraban diseñadas bajo las normativas sismo resistente de aquella época (Tena, 2004), coincidieron en el mismo tipo de falla de entre piso flexible y torsión.

Este evento ocasionó fallas de edificios abiertos, que de acuerdo a la definición propuesta por Aguiar, et al. (2009), es un edificio esquinero cuyas paredes colindan con las estructuras adyacentes, estando acopladas a la estructura, tal como se muestra en la figura 8 , siendo en las columnas de primer piso las solicitadas de mayor forma.

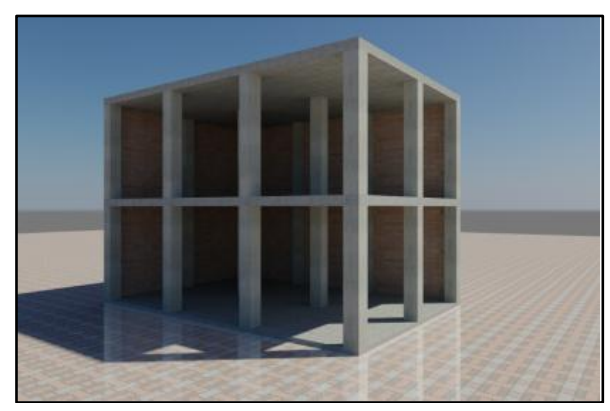

Figura 8. Configuración de edificio abierto

Los daños en los edificios abiertos, se presentan por el efecto de torsión debido a la excentricidad de los planos de pisos y también, porque las columnas son mucho más altas y esbeltas, con el fin de dejar un nivel para ser utilizado como locales comerciales, dándose así la diferencia de rigideces entre pisos.

La falla por piso suave, fue aproximadamente la causa del $9 \%$ de daños en las edificaciones de acuerdo al Instituto de Ingeniería UNAM (1985), presentándose principalmente en edificios destinados a uso multifamiliar, los cuales presentaban notables diferencias en la rigidez, resistencia y ductilidad entre los nivel superiores e inferiores.

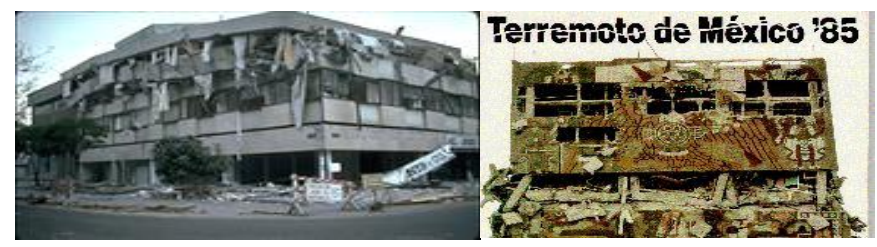

Figura 9. Configuración de edificio abierto 
Es importante destacar, que es la primera planta la que recibe el mayor cortante producto del efecto del terremoto, por lo que el calculista debe diseñar de manera local y global los elementos estructurales, con el fin de que estos absorban las demandas de deformación.

\subsection{Sismo Northrigde, California (1994)}

Sin duda, este sismo marcó claramente un hito en el desarrollo de la ingeniería sismo resistente en los Estados Unidos de América, y por ende en el mundo. Ya que al ser afectada la ciudad de los Ángeles, el gobierno local y las universidades dieron toda su atención a la investigación y desarrollo de acciones de prevención de riesgos, propuesta de nuevas normativas, inversión pública-privada, entre otros factores que influyeron en los avances de los sistemas constructivos y el diseño.

La magnitud del evento fue de 6.7 con una profundidad de $18,3 \mathrm{~km}$, siendo el foco de ruptura en el valle de San Fernando el día 17 de enero 1994. Presentándose gran cantidad de falla de piso débil, tal como se muestra en la figura 10 , donde edificios y viviendas fallaron en la primera planta, entre otros tipos de falla.

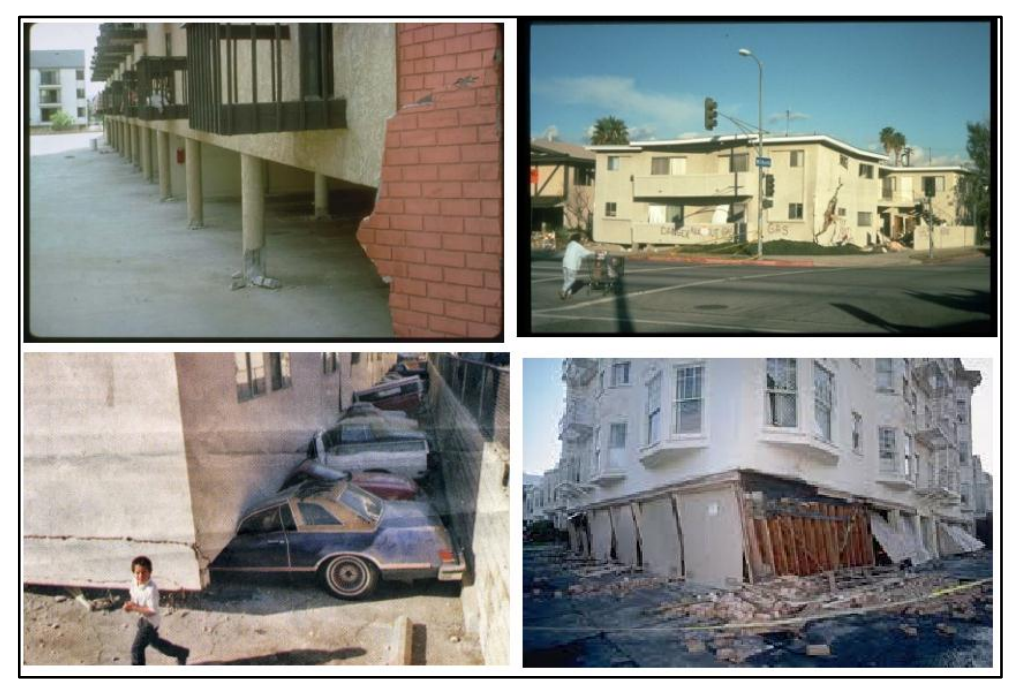

Figura 10. Configuración de edificio abierto

Este tipo de daño estructural, se evidenció en diferentes tipos de materiales como en hormigón, madera y adobe estructural.

\subsection{Sismo de Perú (2007)}

El epicentro de este sismo fue muy cerca de la capital de la ciudad de Chincha Alta, con una profundidad de $8 \mathrm{~km}$ y con un impacto muy fuerte, debido al fallecimiento de 519 habitantes, teniendo su mayor daño en Chincha Alta, Ica y Pisco (EERI 2007). 
Las técnicas constructivas en las ciudades donde el sismo impactó fueron muy similares, siendo principalmente estructuras de mampostería y aporticadas, con el primer nivel muy vulnerable producto de su diferencia de rigidez y resistencia, como el que se muestra en la figura 11.

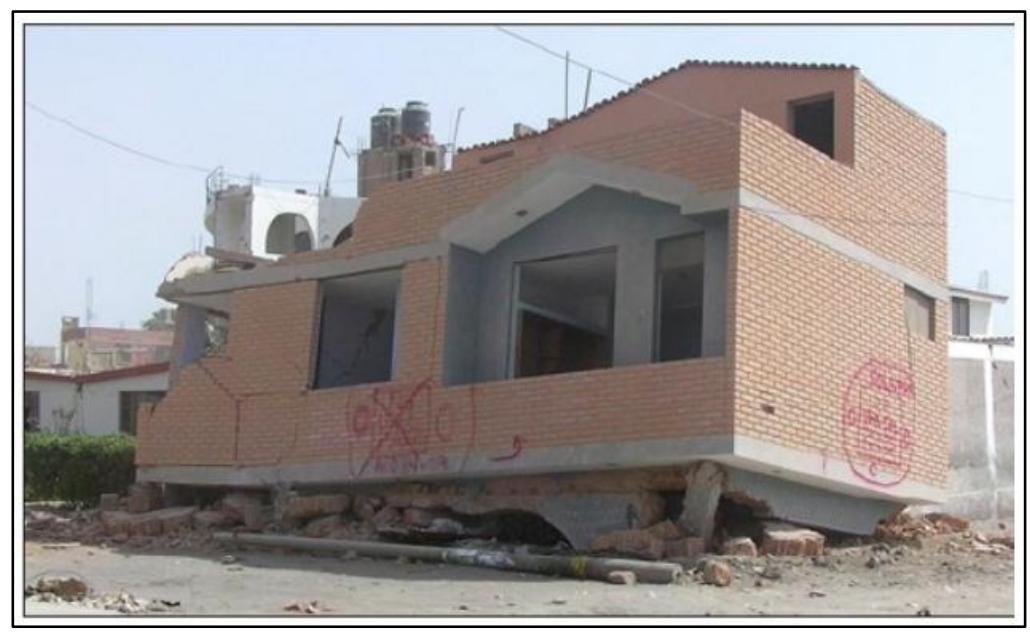

Figura 11. Configuración de edificio abierto

\subsection{Sismo de Puerto Príncipe, Haití (2010)}

Este terremoto ha sido el que mayor vidas humanas ha afectado, dejado más de 235000 muertes y aproximadamente dos millones de damnificados. La magnitud del sismo fue de 7.0 grados con una profundidad de $13 \mathrm{Km}$. Los daños ocasionados por este evento fueron devastadores, tanto en viviendas privadas como en edificaciones estatales, las cuales colapsaron producto del efecto de sitio y la baja calidad constructiva.

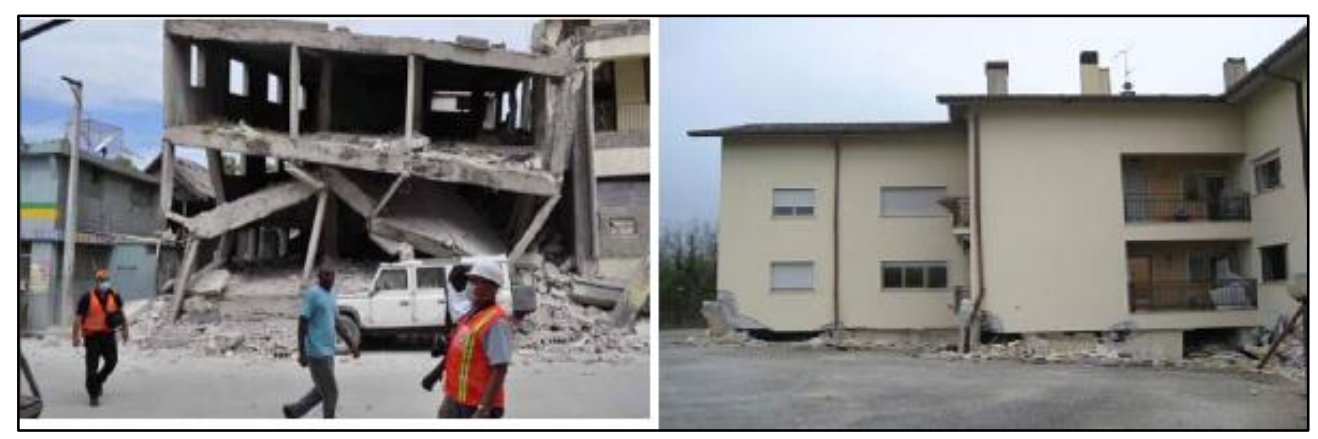

Figura 12. Daño estructural en viviendas

En la figura anterior, se puede evidenciar muchas edificaciones que eran de utilidad multifamiliares, que fallaron por piso débil, siendo esta la causa de colapso que más se presentó, y las pocas que quedaron "en pie" debieron ser demolidas por presentar daños irreparables. 


\subsection{Sismo Maule, Chile (2010)}

En el mega sismo de 2010 que ocurrió en Chile con una magnitud de 8.8 grados afectó la zona costera central, y afecto a países vecinos como Argentina y Perú. A pesar de su magnitud tan grande, los daños que este terremoto causó, no fueron proporcionales a la misma. Originándose muchas hipótesis que buscaban explicar esta situación, con criterios que van desde que la densidad poblacional de este país es muy baja, las normativas de construcción son muy estricticas o que por la frecuencia de los terremotos, hace que estos eventos naturales sean los mejores fiscalizadores de las edificaciones mal construidas.

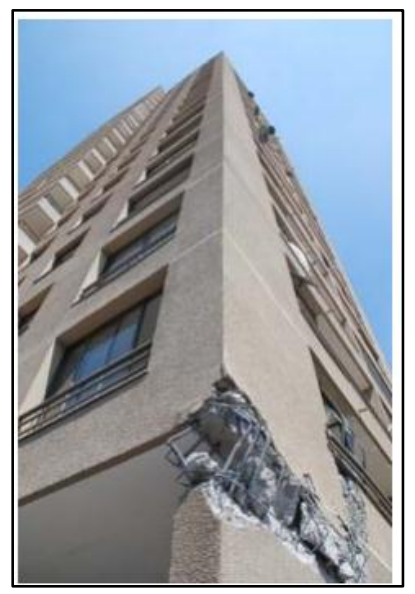

Figura 13. Daño estructural de piso blando con muro de corte

Algo importante sobre este evento fue expresado por Aguiar (2011), quien resalta el hecho de que se encontraron daños de piso débil en estructuras que estaban construidas con muro de corte (diafragmas), tal como se muestra en la figura 13, por lo que este autor indica, que no hay garantía de evitar fallas por piso blando si se construye edificios con muro de corte, ya que este tipo de fallas es ocasionada por la diferencia de rigidez de los pisos.

\section{COMPORTAMIENTO DE ESTRUCTURAS DE PISO BLANDO EN EL TERREMOTO DEL 16 DE ABRIL DE 2016}

Como consecuencia del evento telúrico ocurrido el 16 de abril del 2016 en el Ecuador, se origino el colapso de muchas estructuras y otras quedaron con daños irreversibles, que impidieron aplicar técnicas de reparación, debido a que las patologías presentadas fueron consideradas como muy graves.

En la sección anterior, se mostro como a pesar del paso del tiempo y en diferentes contextos geográficos, las fallas en las edificaciones ocasionadas por eventualidades sísmicas son muy similares, y el daño por piso débil o flexible no es una excepción, por lo contrario, es una muestra a la regla que indica, que las edificaciones que tienen irregularidad en elevación y planta, con pisos débiles, fallan ante el embate de fuerzas sísmicas considerables. 
Las imágenes que se muestran a continuación, demuestran que a pesar de la existencia de suficientes argumentos que indican que este tipo de irregularidades en elevación son muy vulnerables, en las edificaciones del Ecuador, este tipo de configuración es muy común.

La figura 14 presenta el Hotel LUN-FUN, el cual tenía cuatro niveles, siendo el primero destinado para el funcionamiento de un restaurant, nivel que tenía pocas divisiones internas y era de mayor altura que los demás, con lo que se disminuía considerablemente la rigidez en relación a las demás plantas.

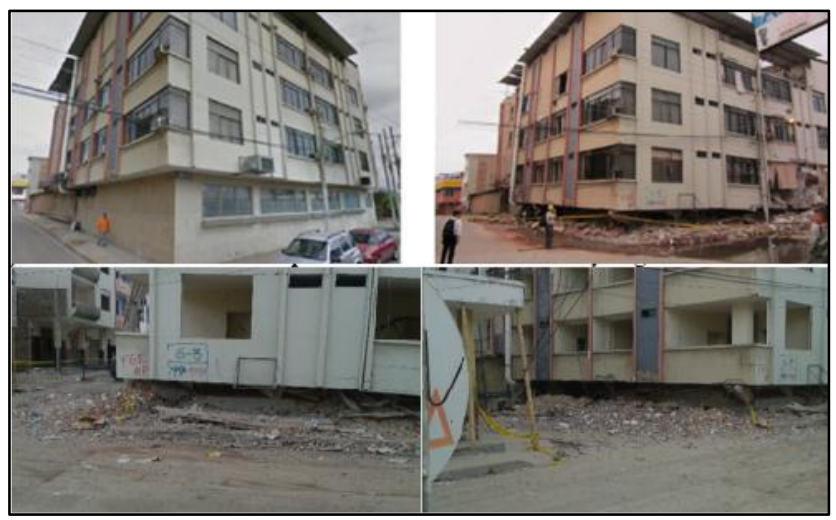

Figura 14. Hotel Lun-Fun en Manta, colapsado por el terremoto

Las viviendas construidas en la zona costera de Ecuador, regularmente presentan una configuración estructural muy vulnerable, ya que a consecuencia de las altas temperaturas, mayormente los primeros niveles presentan alturas diferentes a los otros pisos, lo que hace a la vivienda más flexible y menos estable; sumado a que las construcciones en el país para este tipo de edificaciones es de carácter artesanal, por lo que se ejecutan sin o poco control de calidad, con una inspección o dirección técnica no muy responsable que permita garantizar un desempeño estructural adecuado de las viviendas.

En la figura 16 se puede apreciar una vivienda, donde se nota que las columnas de la planta baja cedieron producto de la falla por piso débil, un volado considerable y un reservorio de agua en el último nivel, elementos que incrementaron el efecto sísmico.

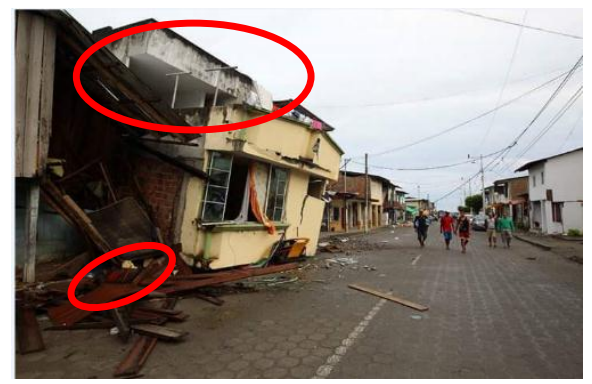

Figura 16. Vivienda colapsada. 
Entre las edificaciones estudiadas, se pudo conocer el principal uso de estos eran departamentos, en la figura 17 se observa un estructura colapsada por la falla en estudio, donde se puede apreciar la diferencia entre los materiales de construcción utilizados, lo que sugiere es que fueron construidos en tiempos diferentes, situación muy típica en la zona. Así como el adozamiento de nuevos niveles en la estructura, que no poseen las características dinámicas para soportar la combinación de las cargas ante eventos sísmicos.

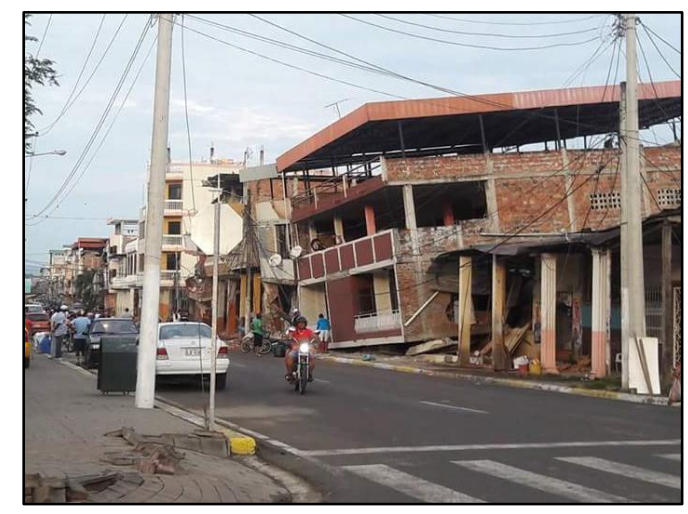

Figura 17. Irregularidad en elevación

El casco comercial de la ciudad de Portoviejo fue otra zona muy afectada, como el caso que se presentó en el edificio de tipo abierto que se muestra en la figura 18, el cual colapsó debido a combinación por falla de torsión y piso débil; además, su diferencia de rigidez en los planos y en los niveles, produjo incremento de esfuerzos en las columnas del primer nivel, y estas no resistieron los efectos de deformación a las que fueron sometidas por sismo.

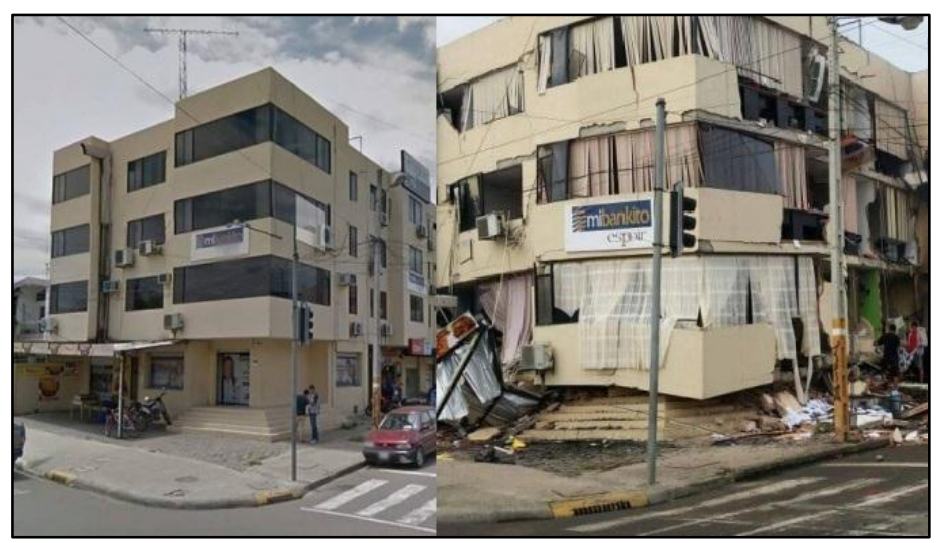

Figura 18. Edificio abierto y piso débil, Portoviejo

Por último, el autor pudo determinar de manera general en ciertas estructuras sometidas a fuerzas sísmicas, después de hacer una comparación entre un antes y después, que las posibles causas de fallas de las edificaciones en el área de Portoviejo, fueron producto de la amplificación de las ondas sísmicas debido al 
tipo del suelo en la zona, lo que ocasionó el colapso de varios edificios, entre éstos el Instituto Ecuatoriano de Seguridad Social y un edificio comercial céntrico, como se puede apreciar en la figura 19 , colapso atribuible a problemas de piso flexible de las edificaciones.

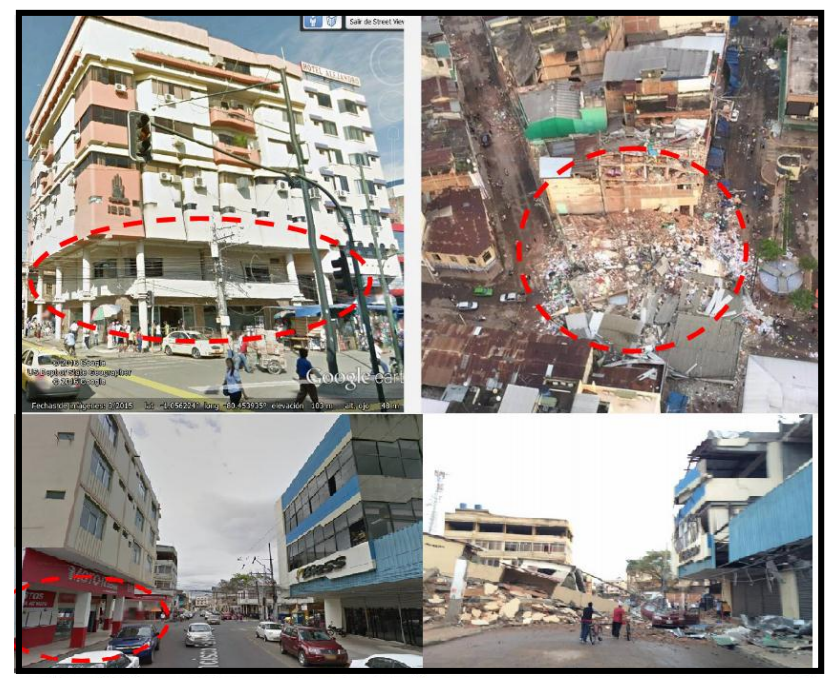

Figura 19. Edificio IESS y comercial, en el centro de Portoviejo

\section{PLANTEAMIENTO DE SOLUCIONES A LA FALLA DE PISO DÉBIL}

En la literatura científica y técnica sobre patología estructural, se pueden encontrar varias propuestas o normativas técnicas que permiten mejorar el desempeño de las edificaciones con piso débil. Además, el personal de investigación que pertenece a la escuela de Ingeniería Civil de la Universidad Laica Eloy Alfaro de Manabí y el Centro de Investigación en Ingeniería Sísmica se encuentran desarrollando varios trabajos, enfocados al análisis de soluciones posibles con el objetivo de mejorar el desempeño de las estructuras a diseñar o existentes que presentan éste aspecto patológico.

Un de los proyectos en ejecución, estudia el reforzamiento en estructuras existentes que presentan falla de piso débil, y plantea sugerencias para futuros diseños, por lo que en este artículo mencionaremos algunas técnicas con una descripción breve de sus ventajas.

- Mejorar la configuración estructural de las edificaciones, evitándose el uso de columnas muy esbeltas y disminuir el cambio brusco de rigideces, reduciría el riesgo de colapso.

- Aumentar la rigidez lateral de la planta débil, mediante el uso de muros estructurales adicionales entre los elementos individuales en el piso blando.

- Construcción de vigas de riostras en los niveles con rigidez menor o en toda la estructura, con la finalidad de disminuir el desarrollo de grandes deformaciones. 
- Disminuir la rigidez en los pisos superiores, a través del aislamiento de los elementos no estructurales, principalmente de la mampostería, que en ocasiones no es considera en el cálculo, pero desde el momento de la etapa constructiva, se integra a los planos estructurales, ocasionando un aumento importante en la rigidez lateral, por lo que se puede utilizar elementos alivianados o aislarlos mediante la aplicación de epóxicos especiales.

- Uso de aisladores y/o disipadores de energía, permitirían mejorar el comportamiento estructural de una edificación, evitando que sea sometida a grandes esfuerzos que la lleven al daño local de los elementos y a su vez a la falla de la estructura.

\section{CONCLUSIONES}

La ingeniería sismo resistente tiene actualmente una responsabilidad muy grande desde el momento en que se proyecta una edificación, y el estudio realizado a la falla por piso débil, nos permitió observar, que aun cuando se ha aprendido mucho de las consecuencias de los eventos sísmicos de grandes magnitudes en diferentes países, todavía las edificaciones siguen construyéndose de manera poco adecuada, interviniendo factores como los cuidados en la construcción, el mantenimiento, la calidad de los materiales, entre otros; y además, el factor de que las técnicas constructivas en los países mencionados en este estudio han variado muy poco. Por último, sólo nos queda esperar que la ingeniería sismo resistente logre convencer a la sociedad, que la solución y prevención a este tipo de desastres, es cambiar las técnicas constructivas, e incorporar el uso de dispositivo aisladores y disipadores de energía que protejan las estructuras del embate de las fuerzas sísmicas.

\section{REFERENCIAS}

1. Aguiar, R. Jara, A. Páez, D (2009), Edificios abiertos con y sin aisladores de base. Revista CIENCIA Vol 12,1-25. Quito- Ecuador

2. Aguiar, Roberto (2011). Fallas frecuentes durante los terremotos . Centro de Investigaciones Científicas -ESPE. Ecuador

3. Álvarez G., Salvador, (1987). Informe Técnico-Sismológico del Terremoto de San Salvador del 10 de Octubre de 1986. Centro de Investigaciones Geotécnicas, San Salvador, El Salvador.

4. Fernández, Lucioano (2007), Efectos de interacción dinámica sueloestructura en edificios con primer piso blando. Pág.2, 2007

5. Instituto de Ingeniería, UNAM (1985). La UNAM ante los sismos del 19 de septiembre de 1985, Informe, México 
6. Kaminetzky, D. (1991). Design and Construction Failures. New York.: McGraw-Hill

7. Padilla, G. (2010). Propuesta para considerar la irregularidad estructural en la resistencia lateral de las estructuras en el Perú (Tesis para optar título). Lima: Pontificia Universidad Católica del Perú.

8. Pujades, Luis (2003). Vulnerabilidad sísmica de edificaciones esenciales. Análisis de su contribución al riesgo sísmico. Tesis doctoral, Universidad Politécnica de Cataluña, España

9. Quincot, G. P. ( 2010 ). Propuesta para considerar la irregularidad estructural en la resistencia lateral de las estructuras en el Perú . Lima.

10. Tena, A (2001), Respuesta sísmica de edificios de concreto reforzado irregulares en esbeltez y en elevación diseñados para distorsiones de entrepiso altas, XIII Congreso Nacional de Ingeniería Sísmica, Guadalajara, CDROM, noviembre.

11. Tena, A (2004), Daños durante sismos recientes y enseñanzas principales, Memorias, II Seminario de Reaseguro Facultativo "Terremoto y Huracán", México, D. F, pp 51-87, septiembre. 